\title{
Identification of HCV Inhibitors from a Cell-Based Sub-Genomic Replicon Screen
}

\author{
David C. Pryde ${ }^{1 *}$, Thien-Duc Tran ${ }^{1}$, Mark Gardner ${ }^{1}$, Chris Pickford $^{2}$, Stephen M. Shaw ${ }^{2}$, \\ Mike Westby $^{2}$, Tanya Parkinson ${ }^{2}$, Caroline Smith-Burchnell ${ }^{2}$, Malcolm Macartney $^{2}$, \\ John H. Tatlock ${ }^{3}$, Rohit Duggal ${ }^{4}$, Leena B. Patel ${ }^{3}$, Tanya M. Jewell ${ }^{3}$, Scott C. Sutton ${ }^{3}$, \\ Tin H. Tran ${ }^{3}$, Weidong Hao ${ }^{3}$, Rob Webster ${ }^{5}$, Satish Dayal ${ }^{5}$ \\ ${ }^{1}$ Worldwide Medicinal Chemistry, Pfizer Global Research and Development, Sandwich, UK \\ ${ }^{2}$ Anti-Infectives Research Unit, Pfizer Global Research and Development, Sandwich, UK \\ ${ }^{3}$ Worldwide Medicinal Chemistry, Pfizer Global Research and Development, San Diego, USA \\ ${ }^{4}$ Anti-Infectives Research Unit, Pfizer Global Research and Development, San Diego, USA \\ ${ }^{5}$ Pharmacokinetics, Dynamics and Metabolism, Pfizer Global Research and Development, Sandwich, UK \\ Email: *David.Pryde@pfizer.com
}

Received January 19, 2013; revised February 21, 2013; accepted February 28, 2013

\begin{abstract}
A high throughput screen of the Pfizer compound collection was carried out using a hepatitis C virus (HCV) genotype 1 b sub-genomic replicon cell line. Those confirmed hits that demonstrated broad spectrum activity without overt cytotoxicity were further evaluated, leading to the identification of a series of pyrrolopyridines with excellent antiviral activity in a fully infectious HCV cell-based assay and pharmacokinetic properties.
\end{abstract}

Keywords: HCV; Replicon; Antiviral; Cell-Based Screen

\section{Introduction}

It is estimated that some $3 \%$ of the world's population is currently infected with hepatitis $\mathrm{C}$ [1] virus (HCV). Approximately $70 \%$ of infected individuals develop a chronic infection a portion of whom go on to develop chronic liver disease [2]. While the rate of new infections has declined sharply in the last two decades, the need for broadly efficacious and well-tolerated therapies remains high. Current standard of care therapy for HCV is a combination of injected pegylated interferon and ribavirin [3], which can eradicate the virus with varying degress of efficacy depending on the genotype of virus the patient is infected with, and other genetic factors. Genotype 1 (gt1), the most prevalent genotype in Europe and the US, responds only modestly to interferon therapy with a sustained virologic response in approximately half of the treated population and the treatment is associated with severe side effects and significant discontinuation rates [4].

$\mathrm{HCV}$ is also a highly mutable virus, and as resistance mutations emerge, new and effective antiviral mechanisms are needed. Thus, there remains a high need for new HCV therapies with improved efficacy and toleration compared to the current standard of care particularly against the more difficult to treat gtl viral population.

The most common therapeutic strategy being pursued

"Corresponding author. within the pharmaceutical industry is to target direct acting antivirals [5]. For example, Vertex's telaprevir 1 [6] and Schering-Plough's boceprevir 2 [7] (Figure 1) were both approved in the US in 2011 and target the NS3 protease, while filibuvir 3 [8] from Pfizer is currently in Phase II trials and targets the NS5B polymerase. Daclatasvir 4 [9] from Bristol-Myers Squibb is also in Phase II and targets the NS5A protein. The pharmacological targets of these antiviral agents represent the most common under clinical investigation, beyond which there are relatively few compounds in clinical trials against alternative targets, either host or viral in natur [10]. In our internal programme, we sought medicinal chemistry starting points against new HCV viral targets that could offer a differentiated efficacy and/or resistance profile compared to standard of care or agents undergoing current clinical testing.

\section{Identifying Novel Antiviral Agents for Treating HCV}

In order to identify novel chemistry starting points for broad-spectrum anti-HCV treatments, we initially ran a full-file high throughput screen of the Pfizer corporate compound file, against a genotype $1 \mathrm{~b}$ HCV sub-genomic replicon screen using a fluorescent read-out in 384-well format [11]. 
Given the multifactorial nature of any activity emerging from such a cell-based screen, a detailed screening sequence (Figure 2) was put in place to triage the hits, to eliminate cytotoxic compounds and those with limited or no spectrum across the two most prevalent genotypes gtla and gt $1 \mathrm{~b}$. The intention was to funnel hits down to a small number of well-characterised, structurally attractive hits. At this stage, hits were categorised according to measurable biochemical activity against NS3 and NS5B, and the remaining actives badged as having "unknown mechanism of action (MOA)". The ultimate goal of the screening campaign was to identify the novel discrete molecular targets of these orphan hits through a combination of virology and chemical biology methods.

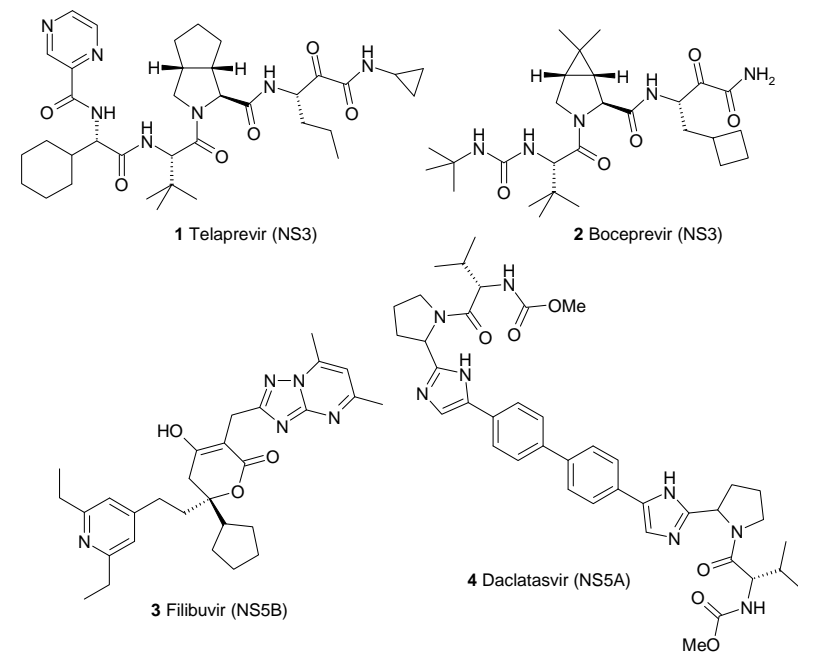

Figure 1. Selected clinically precedented HCV antiviral agents, with their viral target indicated in parentheses.

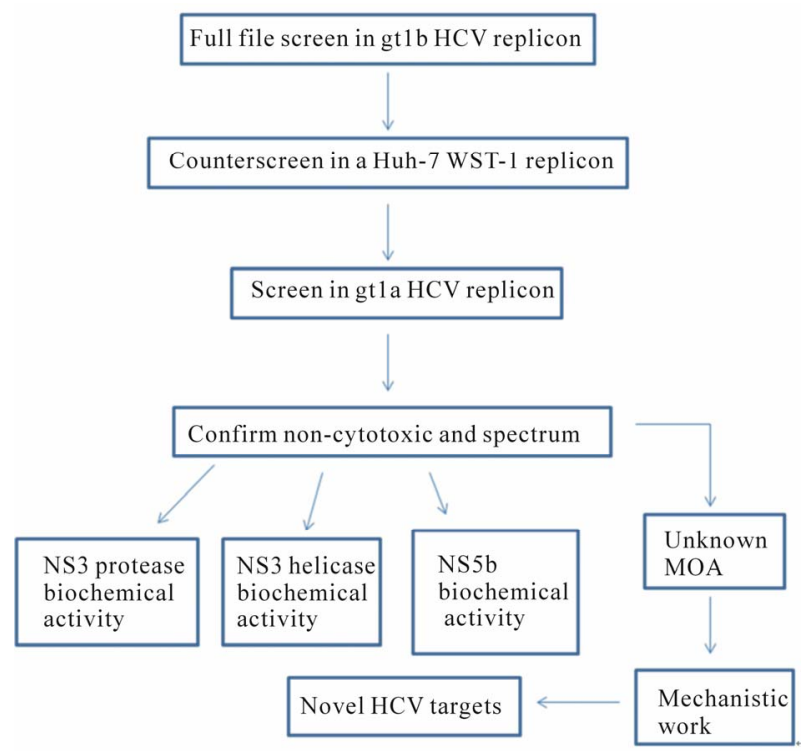

Figure 2. The screening cascade followed to identify novel anti-HCV agents.

\section{Results}

\subsection{Output from a Full File High Throughput Screen}

The initial gt1b screen was carried out using fresh Huh-7 cells hosting the HCV replicon recombined with the luciferase reporter gene [11]. Active compounds in this assay inhibited firefly luciferase expression, a read-out that has been shown to be directly proportional to the level of viral RNA present in a cell. Hits were defined as displaying a greater than 50\% inhibition of luciferase activity at a single test concentration of $10 \mu \mathrm{M}$.

Compounds that passed this criterion had their activity confirmed in a rescreen, and then a cytotoxicity check in a replicon-containing Huh-7 cell line using the WST-1 [12] cell viability reagent to determine viable cell number in response to compound incubation [13]. Activity was then determined against a gtla replicon screen [14]. Further extensive profiling including QC analysis of purity and identity, off-target pharmacology demonstrated by the compounds as well as measured and predicted ADME, toxicity and physicochemical properties was carried out. Following this, three main series were selected as sufficiently interesting for further work, as shown in Figure 3.

The series identified were structurally distinct, possessed low micromolar potency, and had reasonable

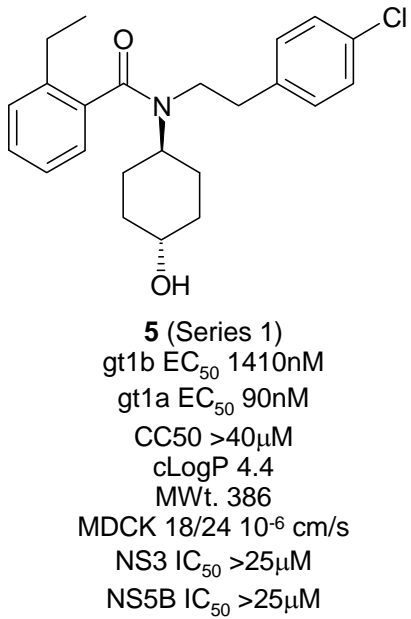<smiles>O=C(NCc1cccs1)c1cc2nc(-c3cccs3)cc(C(F)(F)F)n2n1</smiles><smiles>Cn1c(-c2cccc(O)c2)nc2c(N3CCCCC3)ncnc21</smiles>

6 (Series 2) gt1b EC $\mathrm{E}_{50} 2720 \mathrm{nM}$ gt1a $\mathrm{EC}_{50} 7900 \mathrm{nM}$ CC50 $>40 \mu \mathrm{M}$ cLogP 2.8 MWt. 309 MDCK $5 / 110^{-6} \mathrm{~cm} / \mathrm{s}$ NS3 IC50 $>25 \mu \mathrm{M}$ NS5B IC50 $>25 \mu \mathrm{M}$

7 (Series 3) gt1a $\mathrm{EC}_{50} 5600 \mathrm{nM}$ $\mathrm{CC} 50>100 \mu \mathrm{M}$ CLogP 4.2 MWt. 408 MDCK $8 / 1010^{-6} \mathrm{~cm} / \mathrm{s}$ NS3 IC50 $>25 \mu \mathrm{M}$ NS5B IC50 $>25 \mu \mathrm{M}$ gt1b EC $\mathrm{E}_{50} 4700 \mathrm{nM}$

Figure 3. Primary compound series identified through an HCV replicon high throughput screen. 
physicochemical properties with molecular weights in the region $300-400$ and $\operatorname{cog} \mathrm{P}$ in the region 3 - 4.5. All compounds showed excellent cell permeability, an essential component for our cell based screening cascade. None of the series seed compounds showed any appreciable activity against isolated NS5B or NS3 proteins [15]. Our efforts focussed on developing SAR within each of these series to identify the most robust series to take forward into a full medicinal chemistry programme. For all compounds, gt1b and gtla potency was obtained, along with confirmation in the WST-1 assay that activity was not due to cytotoxicity.

\subsection{Series 1}

Compound 5 emerged as a relatively weak inhibitor of the gt1b replicon, but upon further profiling, a much more potent inhibitor of the gtla replicon, a mismatch in favour of gtla of approximately 15 fold that is quite unusual; gtla activity tends to be more difficult to achieve. When we investigated the screening history of this particular compound, we found that it and close analogues had been pursued as part of a previous programme to find antagonists of the $\mathrm{C} 5 \mathrm{a}$ receptor. Our efforts in this area have been published previously $[16,17]$. This history allowed us to rapidly access several hundred analogues from our compound collection to confirm activity against the HCV replicons. The synthetic methods to add to this existing collection were also well-established and we were able to generate a substantial amount of SAR very quickly. Syntheses of compounds from this series were carried out according to Scheme 1 and is illustated for compound 5.

One of the first aspects of the series we wished to establish was whether replicon activity tracked with C5a activity in which bound $\mathrm{C} 5 \mathrm{a}$ was measured at $4 \mathrm{C}$ using

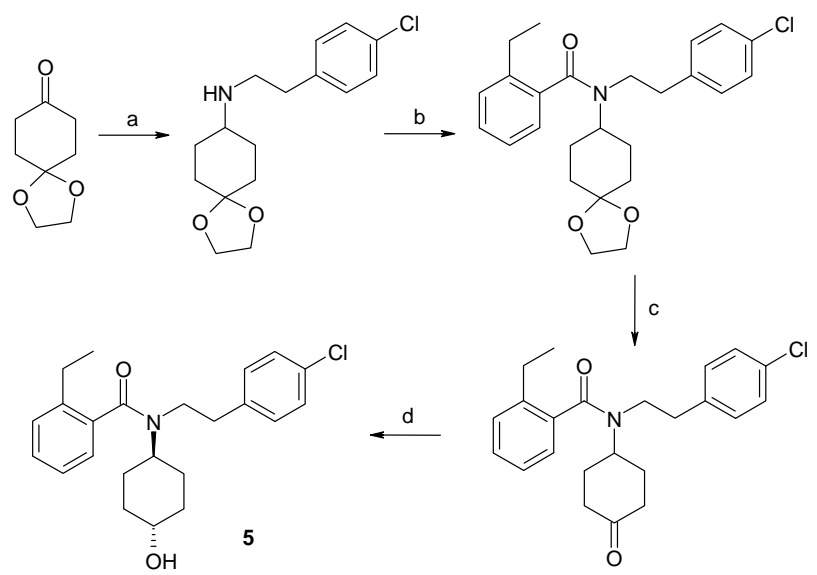

Scheme 1. Preparation of compound 5 from series 1. Reagents and conditions: (a) p-chloro-phenethylamine, $\mathrm{Na}(\mathrm{AcO})_{3} \mathrm{BH}, \mathrm{AcOH}, \mathrm{DCM}, \mathrm{rt}, 18 \mathrm{~h}, 98 \%$; (b) 2-ethylbenzoic acid, $(\mathrm{COCl})_{2}$, DMF, DCM, rt, 1 h, 100\%; (c) $2 \mathrm{~N}$ HCl, THF, $60^{\circ} \mathrm{C}, 3 \mathrm{~h}, 84 \%$; (d) $\mathrm{NaBH}_{4}$, EtOH, rt, 3 h, $78 \%$ (+ approx. 15\% of the corresponding cis diastereoisomer). dibutyryl cAMP-differentiated U937 cells and a filtration assay using 160 pM ${ }^{125}$ I-labelled recombinant human C5a to measure bound radioligand. Compound 5 itself has an $\mathrm{IC}_{50}$ of $428 \mathrm{nM}$ at the $\mathrm{C} 5 \mathrm{a}$ receptor. Using a selection of compounds from the legacy $\mathrm{C} 5 \mathrm{a}$ receptor antagonist programme, Figure 4 demonstrates that there was no correlation between $\mathrm{C} 5 \mathrm{a}$ activity and gt $1 \mathrm{~b}$ replicon activity; the line of unity between the two assays is indicated by the solid line on the chart (there was similarly no correlation with gtla activity-data not shown). Having established that the replicon actvity was not C5a- dependent, we then set out to explore the replicon SAR of the series in more detail.

In Table 1 below, an initial set of compounds is shown in which the synthetically straightforward amide grouping was modified.

Early results suggested that making significant improvements in gtlb activity could be difficult to achieve. Bicyclic systems featuring a lipophilic substituent $(8,9$ and 14) displayed similar gt1b activity, although it is notable that the pyrimidine nucleus in 8 was much weaker against the gtla replicon than a phenyl nucleus. Heterocyclic systems such as 10 and 16 were also weaker against the gtla replicon, while the benzothiophene retained the activity of the starting compound 5 aginst both cell-lines. Replacing the ethyl side-chain in 5 with a chloro substituent in 11 retained activity but replacement of the phenyl nucleus with a cyclohexyl group in 13, or a heterocycle as in 15 , was detrimental to activity.

We then focussed on the phenethyl substituent as shown in Table 2. Relatively conservative changes to the phenyl ring largely retained potency in both replicons, for example the benzofuran 21 . However, when heteroatoms were introduced, for example the benzimidazoles 18 and 19 , the pyrazine 20 , the pyrimidine 26 , triazole 28 ,

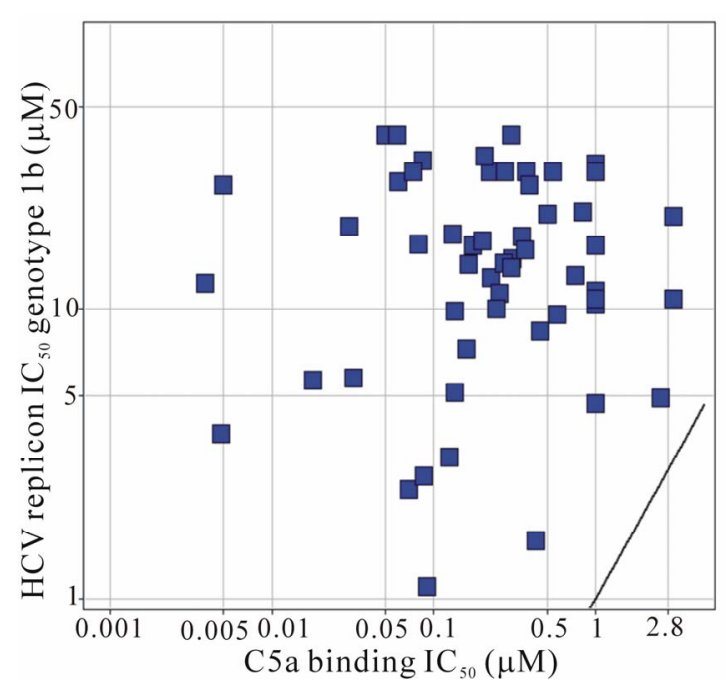

Figure 4. Scatterplot of replicon potency and C5a receptor binding potency. 
Table 1. Amide group structure-activity relationship data within series 1 .

(12)

All figures are in nM. Data is a mean of at least two replicates. Assays are as described in $[11,13,14]$

imidazole 17 or even the closely analogous pyridine 23 , gtla potency in particular suffered. Other more significant structural changes gave similar results, for example the imidazole linked phenyl group in 22, or its fused equivalent 24 were both weak inhibitors of the gt1a replicon. Linking through the pyrazole ring of 25 , or even the cyclohexyl ring of 27 were weak inhibitors of both replicons.

The final area we probed in this initial SAR exercise was in the region of the cyclohexyl alcohol group (Figure 5). For example, the difluoro-cyclohexyl group in 29 was much less active than the cyclohexyl alcohol of compound 5. Basic groups as in 30, extended lipophilic groups as in the trifluoromethyl-substituted cyclohexyl 31 , and even conservative, close analogues such as the methyl ether 32 and the sulfoxide 33 were no better than micromolar active against both replicons.

For the collection of compounds screened and/or made in this series, we had been unable to identify sub-500nM inhibitors of both replicon genotypes. A further concern within the series was that the most potent compounds
Table 2. Phenethyl group structure-activity relationship data within series 1 .

(19)

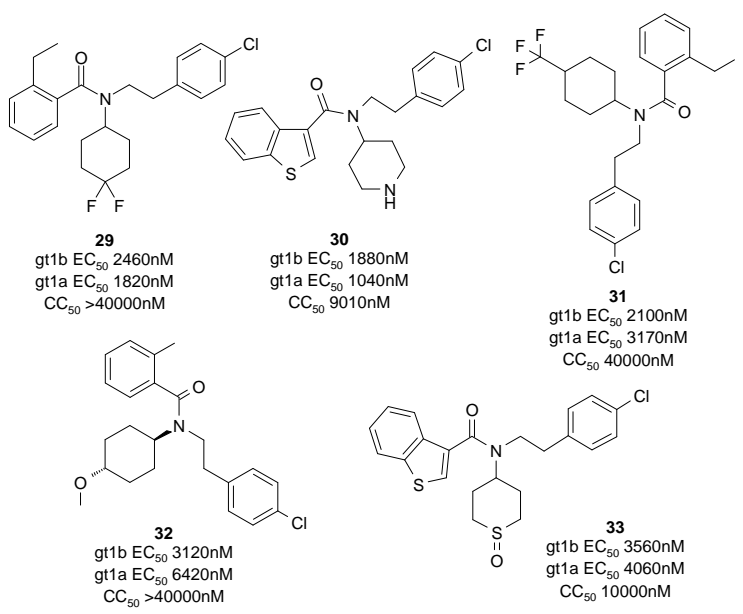

Figure 5. Cyclohexyl alcohol derivatives of series 1. 
appeared to be among the more lipophilic. Attempts to reduce lipophilicity met with only limited success. The Lipophilic Lipoidal Efficiency (LLE or LipE) [18] of the more potent compounds in the series was approximately 4. Within the series, compounds with $\operatorname{cLogP}$ greater than 2 were rapidly turned over in human liver microsomes. Taken together, the inability to find potent compounds against both genotypes, and the rapid in vitro metabolism of compounds potent against gtla, led to the decision to abandon this series.

\subsection{Series 2}

The purine series represented by 6 was an initially weak starting point, albeit balanced against the gtla and gtlb replicons. Compound 6 was synthesised acording to Scheme 2.

Our early SAR investigations initially focussed on the 6-position of the purine core, and examined simple amine substituents in replacement of the piperidine group (Table 3). Growing the size of the substituent to the pyrazinepiperazine 34 , the phenyl pyrazole 35 or the analogous pyrazole-phenyl 36 and its isomer 37 resulted in essentially equivalent potency. The saturated substituents 38 and 39 were weaker in the primary assay, and pyrazole substituents in which the substituent was significantly reduced in size down to just a methyl group in 40 was weaker. When the pyrazole substituent was extended out to a benzyl group in 41, a dramatic increase in activity was observed, in both gtlb and gtla replicons, however this was subsequently confirmed to be due to cytotoxicity.

We devoted some time and a small number of analogues to exploring the cytotoxicity of the benzyl pyrazole analogue 41 through modification of the phenol group (Table 4). Aromatic groups such as the methoxy-phenyl 43 , the cyanophenyl 42 or the pyridine 45 were all notably more cytotoxic than 41.5 -membered heterocycles 47 , or saturated heterocycles 46 maintained essentially no therapeutic index, a similar situation to the contracted

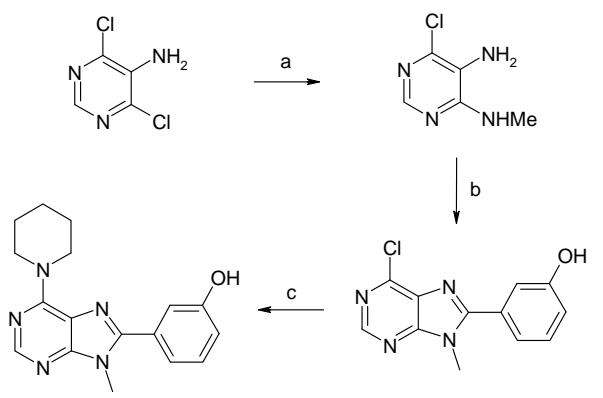

Scheme 2. Preparation of compound 6 from Series 2. Reagents and conditions: (a) Methylamine, diisopropy lethylamine, n-butanol, $120^{\circ} \mathrm{C}, 16 \mathrm{~h}, 100 \%$; (b) m-hydroxy-benzaldehyde, acetic acid, ferric chloride, ethanol, rt, 48\%; (c) Piperidine, triethylamine, DMSO, $150{ }^{\circ} \mathrm{C}$ microwave irradiation, $30 \mathrm{~min}, \mathbf{7 8 \%}$.
Table 3. C-6 analogues of the Series 2 purine lead.

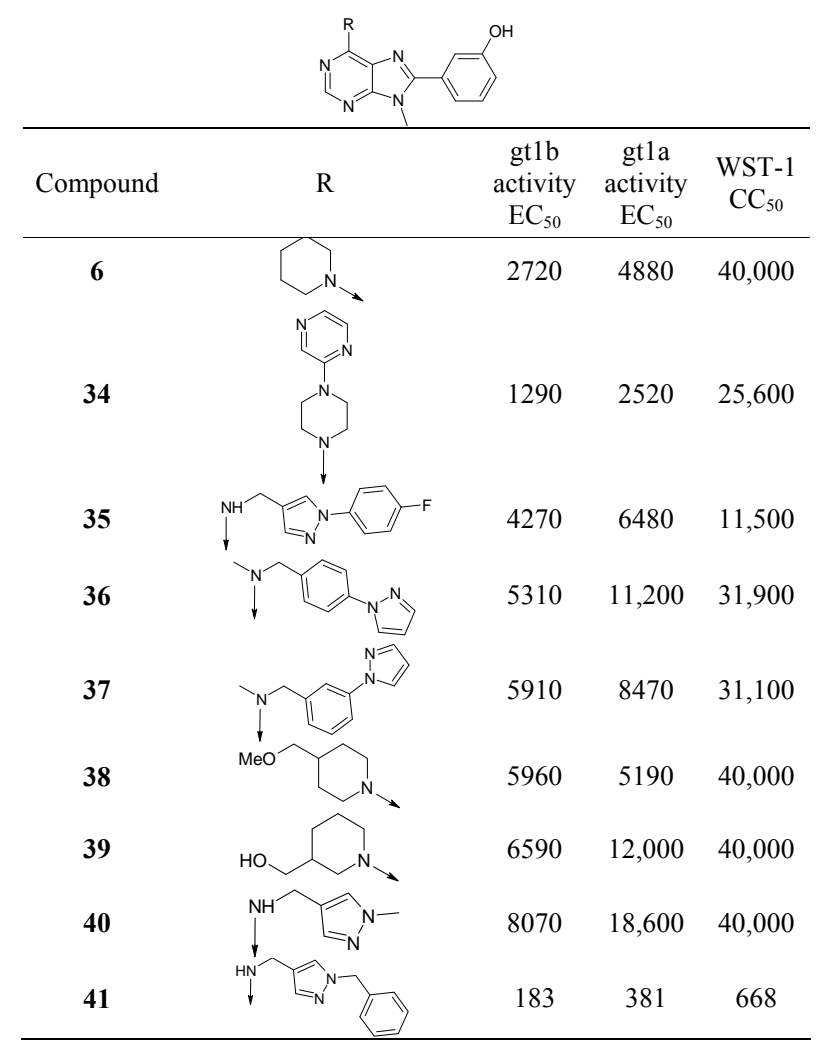

All figures are in nM. Data is a mean of at least two replicates. Assays are as described in $[11,13,14]$.

Table 4. Phenol analogues of the purine-based Series 2.

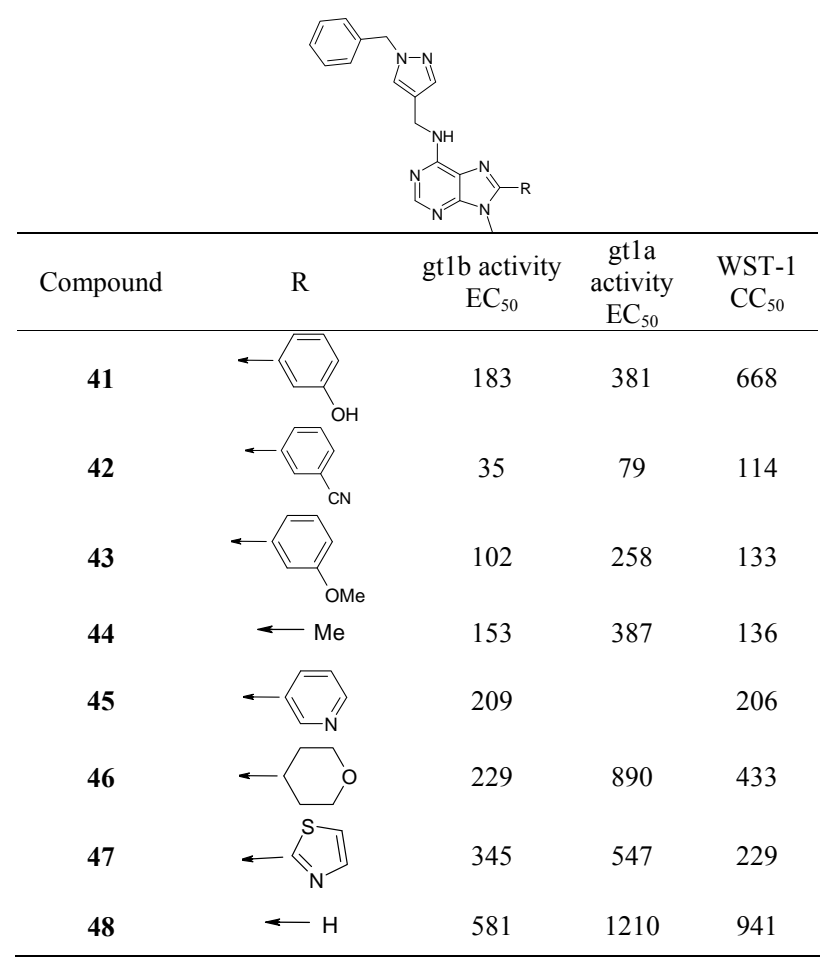

All figures are in nM. Data is a mean of at least two replicates. Assays are as described in $[11,13,14]$. 
methyl group in 44 or a simple hydrogen atom in 48 . These data confirmed that the benzyl-pyrazole purine core carried inherent cytotoxicity through an unknown mechanism.

As a final effort in this series, we were intrigued to see if small changes to the purine core would also maintain cytotoxicity (Table 5). Simple nitrogen atom deletion to give the 1-deazapurine group in 49 gave significantly weaker activity, which again appeared to be due to cytotoxicity. The analogous 3-deazapurine in 50 showed extremely high potency in the WST-1 assay, highlighting how subtle the cytotoxicity effects in this chemotype were. Substructure searching around the purine core uncovered several analogues such as the imidazopyrazine 51 which again showed potent cellular toxicity. More significant structural changes to a quinoline 52 or quinazoline 53 core were required to see drop-offs in cytotoxicity, accompanied by much weaker activity in the primary replicon assays.

At this stage, we were satisfied that this series offered no viable opportunities for a chemistry programme and all futher work was stopped in this chemotype.

Table 5. Purine analogues based on the Series 2 lead structure.

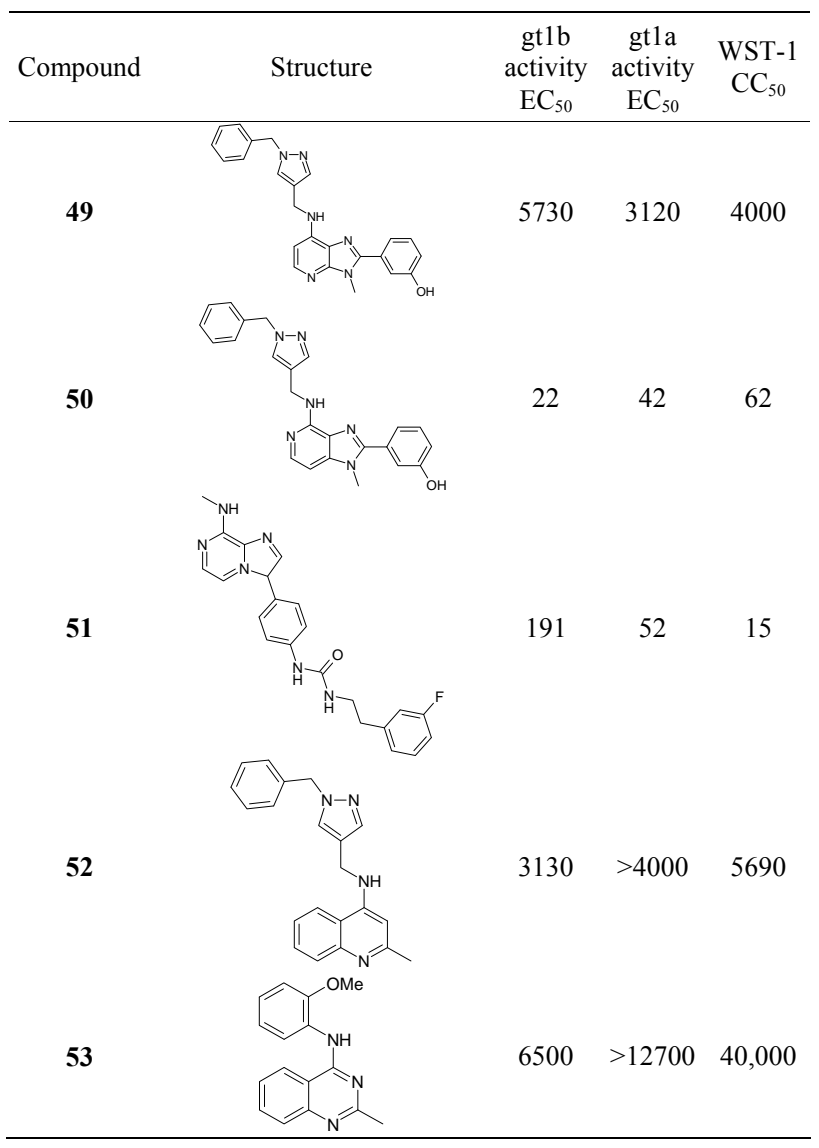

All figures are in nM. Data is a mean of at least two replicates. Assays are as described in $[11,13,14]$.

\subsection{Series 3}

The pyrazolopyrimidine 7 emerged from the high throughput screen as a promising hit, with reasonably balanced, albeit weak cell-based activity. This hit originated from a commercially available compound set $[19,20]$ and contained potentially reactive thiophene rings, which could be readily substituted out, but in normal handling was observed to have poor solubility. It is notable that this structure also bears significant resemblance to similar compounds proposed to be inhibitors of HCV NS4B from Viropharma Inc. based on data from a cell proliferation assay [21]. A substructure search of the Pfizer compound collection uncovered a range of similar compounds featuring diverse core structures 54 - 57, depicted in Figure 6 below. Of these, attention was immediately focussed on the pyrrolopyridine core of 56, which possessed better gtlb potency, while retaining spectrum against the gtla replicon, compared to the starting pyrazolopyrimidine.

Synthetic efforts quickly moved towards expanding the SAR around this core, through modifications to the aryl group pendant to the pyridine ring, and to the amide grouping using the chemistry described in Scheme 3.

Initial data suggested that a lipophilic group at the 6position, adjacent to the pyridine ring of the pyrrolopyridine core, would be essential for high potency. Groups such as tert-Bu, phenyl, substituted phenyl or a methyl-cyclopropyl group did indeed support high potency (Table 6) while polar functionality, for example the alcohol 70, ablated all activity. On the opposite side of the molecule, amide substituents of quite diverse character such as the cyclohexyl alcohol in 58, or the cyclopropyl and cyclopentyl funtionalised alcohols in 60 and 63 respectively were well-tolerated, and while the gtla activity of the series was inferior to their gtlb potency, these were good starting points in an encouraging region of physicochemical space for further optimisation. When these alcohol-containing groups were altered to incorporate further expressions, for example the substituted amino-ethanol unit of 68 , although primary potency against the gt $1 \mathrm{~b}$ replicon declined, this compound was a more balanced starting point against the gtla replicon.

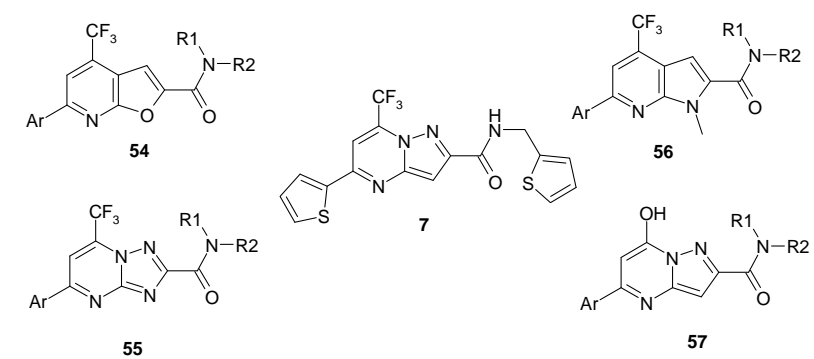

Figure 6. Core variations explored around the pyrazolopyrimidine lead 7 . 


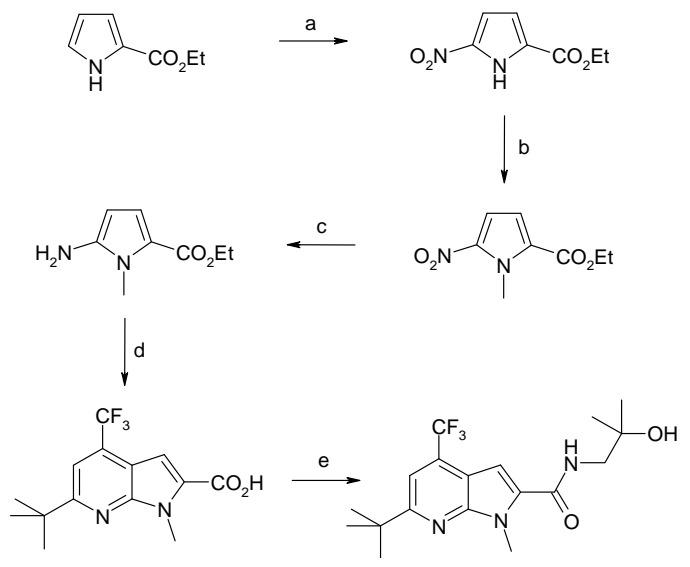

Scheme 3. Preparation of compound 77 from series 3. Reagents and conditions: (a) Acetic anhydride, $\mathrm{HNO}_{3}$, $-78^{\circ} \mathrm{C} \rightarrow$ rt, $16 \mathrm{~h}, 41 \%$. (b) $\mathrm{Cs}_{2} \mathrm{CO}_{3}$, DMF, MeI, rt, $16 \mathrm{~h}$, 41\%; (c) EtOAc, $10 \%$ Pd; (c) 50 bar $\mathrm{H}_{2}$ pressure, $60^{\circ} \mathrm{C}, 16$ h, $93 \%$; (d) Acetic acid, microwave irradiation, $120{ }^{\circ} \mathrm{C}, 15$ min followed by MeOH, LiOH, $50^{\circ} \mathrm{C}, 3 \mathrm{~h}, 88 \%$ over 2 steps; (e) 1-amino-2-methyl-propan-2-ol, 1-propane-phosphonic acid cyclic anhydride, Et3N, DMF, rt, 16 h, $98 \%$.

Table 6. Amide analogues based on the Series 3 pyrrolopyridine structure.

Compound

All figures are in $\mathrm{nM}$. Data is a mean of at least two replicates. Assays are as described in $[11,13,14]$..
Heterocycles exemplified by the tetrahydrofuran in 59 , the piperidine amide in 61 , the sulfones in 65 and 66 and the oxazole in 69 all retained sub- $1 \mu \mathrm{M}$ activity in the gt $1 \mathrm{~b}$ replicon assay. Contracting the amide group down to just a methyl group in 64 or a propyl group in 67 were all approximately micromolar potency. We were encouraged that a basic group in the piperidine unit 62 retained significant activity against the gt $1 \mathrm{~b}$ replicon.

It was encouraging that none of the initial set of analogues appeared to have significant cytotoxicity concerns, but we were concerned that the majority of examples appeared to have a genotype disconnect in favour of gtlb. For this reason, we selected the balanced analogue 68 as the starting point for further structural modification of the core template, described in Table 7. In the 6-position, small changes to the starting trifluoromethyl group, for example through modification to chloro-difluoro in compounds 72 and 73 , or difluoromethyl as in 71 offered modest gains in activity. Bulking

Table 7. Core substitution variations around the pyrrolopyridine template.

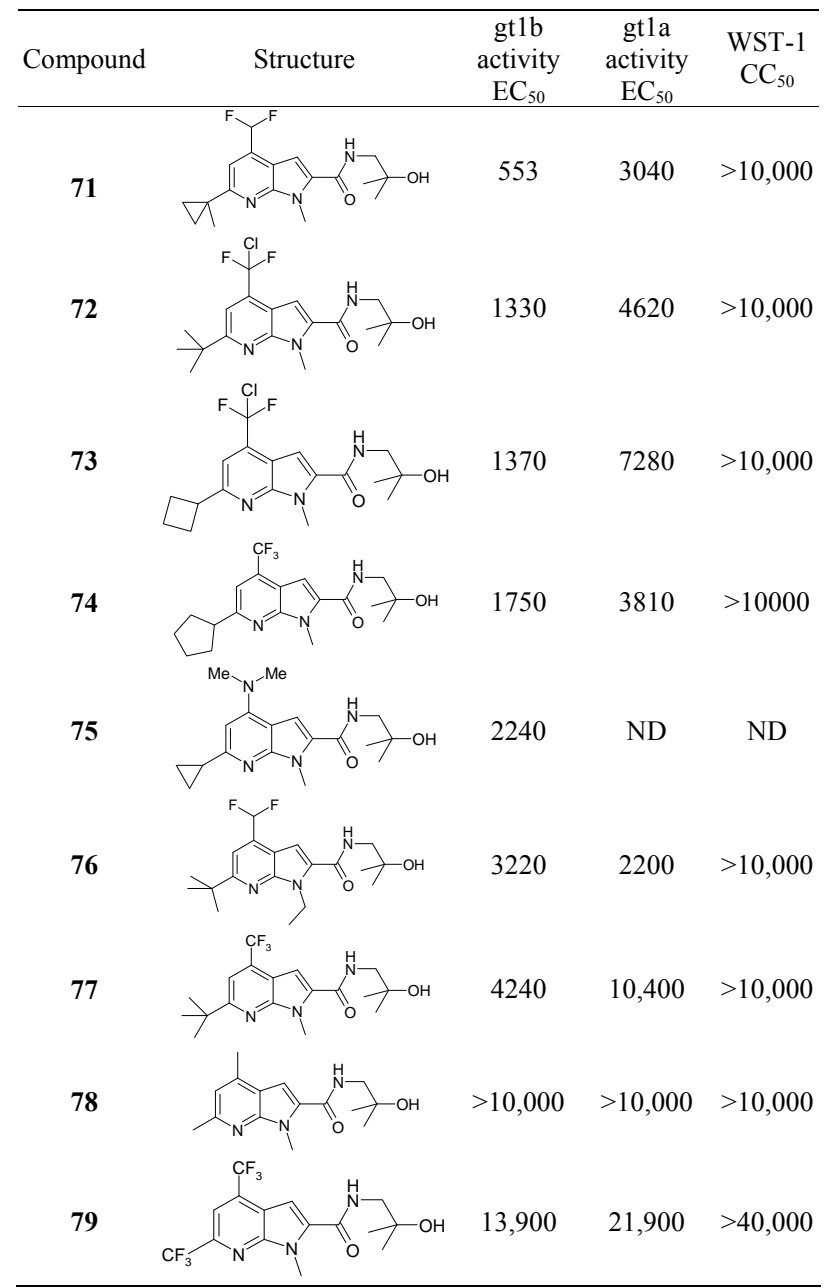

All figures are in $\mathrm{nM}$. Data is a mean of at least two replicates. Assays are as described in $[11,13,14]$. 
up the 2-position group to, for example, a cyclopentyl unit as in 74 again offered a very modest potency gain. An $\mathrm{N}, \mathrm{N}$-dimethyl group in 75 or a homologated core template as in the ethyl-analogue 76 were essentially equipotent.

It also became clear that the combination of the $\mathrm{C} 2$ and C6 groups were critical to the observed activity and genotype coverage. A simple change from the methyl cyclopropyl group of 68 to a tert-butyl in 77 lost gtla potency. Changing to the synthetically simpler dimethyl analogue 78 was inactive against both genotypes, while the bis-trifluoromethyl 79 was somewhat weaker, but retained the balanced genotype activity. At this point, we were faced with what appeared to be quite flat SAR, and little prospect of being able to rapidly analogue into significantly more potent compounds. The physiccochemistry space occupied by compounds from this series was however attractive, with molecular weights in the 400 region and measured lipophilicity values in the range 1.5 - 4. Additional in vitro data was obtained on a selection of compounds from the pyrrolopyridine series, including potency in a fully infectious HCV cell culture (HCVcc) assay [22] which incorporated not just the non-structural proteins of $\mathrm{HCV}$ but also the structural viral proteins that support viral infectivity (Table 8).

The basic group in 62 introduced a significant hERG liability, and the most lipophilic example 60 carried the most human liver microsome turnover, with the intermediate lipophilicity examples 77 and 79 showing moderate microsomal stability and the polar sulfone 66 showing good stability.

The most interesting piece of data we obtained on this subset of compounds was the HCVcc activity in comparison to the replicon assay data. The pyrrolopyridine compounds were consistently much more potent in the HCVcc assay than the replicon assay, and when we subsequently profiled compounds from the other series investigated in the project, a finding which contrasted with all other series (Figure 7).

The infectious assay is a different genotype (gt2a) compared to the replicon assays we routinely used (gtlb and gt1a), and we could not rule out the possibility that the difference between replicon and infectious assay potency was not due to genotype difference as we did not have access to a gt 2 a replicon. The most difficult to treat $\mathrm{HCV}$ genotypes are of gt1, and this was the primary target we set out to inhibit. Alternatively, it is also possible that the azaindole series interacted with a target in the HCVcc system that was not represented in the replicon cell assay, and that the mismatch between replicon and HCVcc potencies would translate across genotypes. An important question became whether translation to a human infection would scale from the replicon or from the infectious in vitro assay potency values.

Taking compound 77 as an exemplar, rat pharmacokinetics were obtained as shown in Table 9. Predictions of human pharmacokinetics were made by scaling from the rat and dose predictions then made using the infectious assay data.

Taking the efficacious target concentration for 77 as the infectious assay $\mathrm{IC}_{50}$ of $46 \mathrm{nM}$, it was predicted that

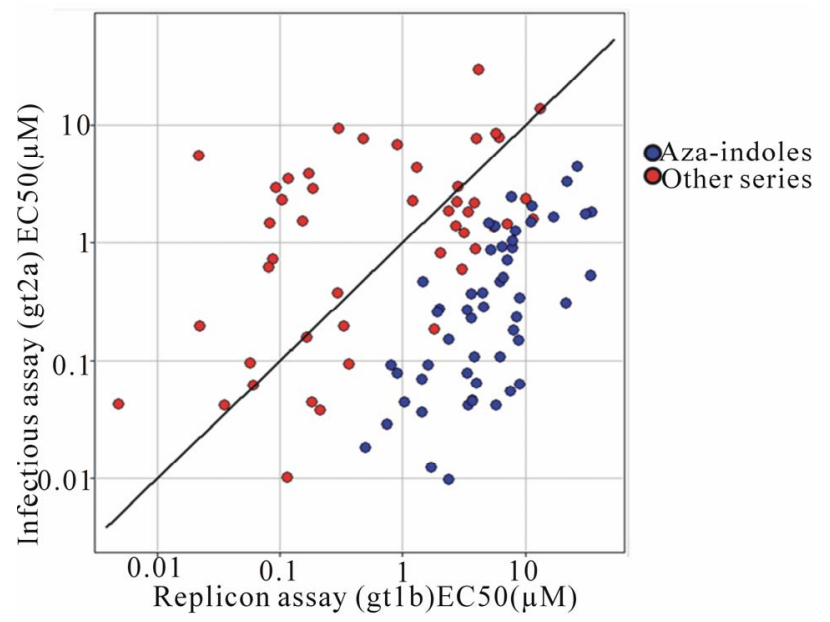

Figure 7. Infectious assay potency (gt2a) vs replicon potency (gt1b) for compounds of the azaindole series compared to those from other series.

Table 8. In vitro potency and ADMET data for selected pyrrolopyridines.

\begin{tabular}{|c|c|c|c|c|c|c|c|}
\hline Compound (MWt.) & $\begin{array}{l}\text { gt1b replicon } \\
\text { IC } 50, \mathrm{nM}^{\mathrm{a}}\end{array}$ & $\begin{array}{l}\text { gt1a replicon } \\
\text { IC } 50, \mathrm{nM}^{\mathrm{a}}\end{array}$ & $\begin{array}{l}\text { gt2a HCVcc } \\
\text { IC50, nM }\end{array}$ & $\begin{array}{c}\mathrm{MDCK}^{\mathrm{c}} \mathrm{AB} / \mathrm{BA}, \\
10^{-6} \mathrm{~cm} / \mathrm{s}\end{array}$ & $\operatorname{cLog} \mathrm{P}\left(\log \mathrm{D}^{\mathrm{d}}\right)$ & $\begin{array}{l}\mathrm{HLM}, \\
1 / \mathrm{min} / \mathrm{mg}^{\mathrm{e}}\end{array}$ & $\begin{array}{l}\text { Dof. Binding } \\
{\text { IC } 50, \mathrm{nM}^{\mathrm{f}}}\end{array}$ \\
\hline $60(383)$ & 1370 & 2470 & NT & $2 / 4$ & $3.2(3.8)$ & 52 & $>20,000$ \\
\hline $62(410)$ & 2460 & 4060 & 10 & $0.1 / 1$ & $4.4(1.8)$ & 20 & 1230 \\
\hline $66(429)$ & 4300 & 16,700 & 29 & $5 / 8$ & $1.6(\mathrm{ND})$ & 9 & $>40,000$ \\
\hline $77(371)$ & 4240 & 10,400 & 46 & $7 / 11$ & $3.8(3.1)$ & 41 & 25,600 \\
\hline $79(383)$ & 13,900 & 21,900 & 916 & $24 / 24$ & $2.9(3.6)$ & 10 & $>20,000$ \\
\hline
\end{tabular}

All figures are in nM. Data is a mean of at least two replicates. ${ }^{a}$ Replicon assays are as described in [11] and [14]. ${ }^{\mathrm{b}}$ The HCVcc assay is described in [22]. ${ }^{\mathrm{c}}$ Methodological details for the MDCK permeability assay have been published previously [23]. ${ }^{\mathrm{d}} \mathrm{LogD}$ measured at pH7.4 in octanol/neutral buffer. ${ }^{\mathrm{e}}$ Details of the HLM assay used have been published previously [24]. ${ }^{\mathrm{f}}$ Details of the hERG assay used, in which compounds are assessed for their ability to displace labelled dofetilide has been reported previously [25]. NT = not tested. 
Table 9. Rat pharmacokinetics and predicted human pharmacokinetics for compound 77.

\begin{tabular}{cccccc}
\hline & Dose & $\mathrm{Cl}(\mathrm{mL} / \mathrm{min} / \mathrm{kg})$ & $\mathrm{Vdss}(\mathrm{L} / \mathrm{kg})$ & $\mathrm{f}_{\mathrm{u}}$ & $\mathrm{F}(\%)$ \\
\hline Rat pharmacokinetics & $2 \mathrm{mg} / \mathrm{kg}$ & 39 & 5 & 0.007 & ND \\
$\begin{array}{c}\text { Human predictions to achieve } \mathrm{C}_{\mathrm{av}} \\
\text { above IC } \mathrm{IC}_{50} \text { concentration at trough }\end{array}$ & $100 \mathrm{mg} \mathrm{bid}$ & 0.8 & 5 & 0.01 & $\sim 90$ \\
\hline
\end{tabular}

$\mathrm{ND}=$ not determined.

a $100 \mathrm{mg}$ bid dose of this compound would provide adequate coverage at steady state. While this constitutes a reasonable dose prediction, any predictions based on the replicon $\mathrm{IC}_{50}$ value would clearly give an unnacceptably high dose. Further in vivo profiling to allow deconvolution of these very different scenarios is yet to be carried out.

\section{Conclusion}

We have described a full file high throughput screen of the Pfizer corporate compound collection using a subgenomic HCV replicon cell-based screen to identify several attractive starting points for medicinal chemistry follow-up. Through hit expansion and SAR activities, a series of azaindoles were prioritised as being most likely to deliver a useful clinical profile, particularly based on their high potency in a fully infectious HCV cell screen.

\section{Acknowledgements}

D. C. P. thanks LesaWatson, Fiona Adam, Gemma Parsons and Nick Smith for their help in synthesis of samples, and Mel Glossop for her help in preparing this manuscript.

\section{REFERENCES}

[1] D. Lavanchy, "The Global Burden of Hepatitis C," Liver International, Vol. 29, Suppl. 1, 2009, pp. 74-81. doi:10.1111/j.1478-3231.2008.01934.x

[2] R. S. Brown, "Hepatitis C and Liver Transplantation," Nature, Vol. 436, No. 7035, 2005, pp. 973-978. doi:10.1038/nature04083

[3] J. H. Hoofnagle and L. B. Seeff, "Targeting the NonStructural Proteins of Hepatitis C Virus: Beyond Hepatitis C Virus Protease and Polymerase," The New England Journal of Medicine, Vol. 355, 2006, pp. 2444-2451. doi:10.1056/NEJMct061675

[4] S. J. Hadziyannis, H. Sette, T. R. Morgan, V. Balan, M. Diago, P. Marcellin, G. Ramadori, H. Bodenheimer, D. Bernstein, M. Rizzetto, S. Zeuzem, P. J. Pockros, A. Lin and A. M. Ackrill, "Peginterferon- $\alpha 2 \mathrm{a}$ and Ribavirin Combination Therapy in Chronic Hepatitis C: A Randomized Study of Treatment Duration and Ribavirin Dose," Annals of Internal Medicine, Vol. 140, No. 5, 2004, pp. 346355.

[5] S. M. Lemon, J. A. McKeating, T. Pietschmann, T. L. Tellinghuisen, D. N. Frick, J. S. Glenn, J. Symons and P.
A. Furman, "Development of Novel Therapies for Hepatitis C," Antiviral Research, Vol. 86, No. 1, 2010, pp. 7992. doi:10.1016/j.antiviral.2010.02.003

[6] L. S. Smith, M. Nelson, S. Naik and J. Woten, "Telaprevir: An NS3/4A Protease Inhibitor for the Treatment of Chronic Hepatitis C," The Annals of Pharmacotherapy, Vol. 45, No. 5, 2011, pp. 639-648. doi:10.1345/aph.1P430

[7] K. X. Chen and G. F. Njoroge, " $1-$ The Journey to the Discovery of Boceprevir: An NS3-NS4 HCV Protease Inhibitor for the Treatment of Chronic Hepatitis C," Progress in Medicinal Chemistry, Vol. 49, 2010, pp. 1-36. doi:10.1016/S0079-6468(10)49001-3

[8] P. L. Beaulieu, "Filibuvir, a Non-Nucleoside NS5B Polymerase Inhibitor for the Potential Oral Treatment of Chronic HCV Infection," IDrugs, Vol. 13, No. 12, 2010, pp. 938-948.

[9] R. A. Fridell, D. Qiu, L. Valera, C. Wang, R. E. Rose and M. Gao, "Distinct Functions of NS5A in Hepatitis C Virus RNA Replication Uncovered by Studies with the NS5A Inhibitor BMS-790052," Journal of Virology, Vol. 85, No. 14, 2011, pp. 7312-7320. doi:10.1128/JVI.00253-11

[10] T. P. Holler, T. Parkinson and D. C. Pryde, "Targeting the Non-Structural Proteins of Hepatitis C Virus: Beyond Hepatitis C Virus Protease and Polymerase," Expert Opinion on Drug Discovery, Vol. 4, No. 3, 2009, pp. 293314. doi:10.1517/17460440902762802

[11] Genotype 1b (Con1, Licensed from Reblikon $\mathrm{GmbH}$ ) HCV Replicon Cells Were Resuspended to a Concentration of $1.4 \times 10^{5}$ Cells $/ \mathrm{ml}$ by Addition of Pre-Warmed Medium (DMEM $+10 \%$ FCS). $45 \mu 1$ of This Suspension Was Added to Each Well a 384-Well Assay Plate (Lumitrac, Greiner) Already Containing 0.51 of Test Compound. All Plates Were Covered with gas Permeable Seals and Incubated at $37^{\circ} \mathrm{C}, 5 \% \mathrm{CO}_{2}$ for 48 Hours. After 48 Hours, the Plate Was Removed from the Incubator and Left to Cool to Room Temperature for 15 - 30 mins. An Equal Volume of Reconstituted Lyophilised Britelite plus Substrate (PerkinElmer) to Medium Was Added to Each Well. Luminescence Was Read Immediately on an EnVision (Perkin Elmer) Plate Reader.

[12] 1[2-(4-Iodophenyl)-3-(4-Nitrophenyl)-5-(2,4-Disulfophen yl)-2H-Tetrazolium], CAS Registry Number, 150849-528.

[13] Genotype 1b (Con1) HCV Replicon Cells Were Resuspended to a Concentration of $1.4 \times 10^{5} \mathrm{cell} / \mathrm{s} / \mathrm{ml}$ by Addition of Pre-Warmed Medium (DMEM +10\% FCS). $45 \mu 1$ of This Suspension Was Added to Each Well of a 384Well Assay Plate (Greiner) Already Containing 0.51 of 
Test Compound. All Plates Were Covered with Gas Permeable Seals and Incubated at $37^{\circ} \mathrm{C}, 5 \% \mathrm{CO}_{2}$ for 48 Hours. After 48 Hours, $5 \mu$ of WST-1 Cell Proliferation Reagent (Roche) Was Added to Each Well and the Plate Returned to the Incubator for 1 Hour. After This Incubation Period Absorbance Was Read at $450 \mathrm{~nm}$ on an EnVision (Perkin Elmer) Plate Reader.

[14] Genotype 1a (H77, Licensed from Apath LLC) HCV Replicon Cells Were Resuspended to a Concentration of $1.4 \times 10^{5}$ Cells $/ \mathrm{ml}$ by Addition of Pre-Warmed Assay Medium (DMEM $+10 \%$ FCS). $45 \mu 1$ of This Suspension Was Added to Each Well of a 384-Well Assay Plate (Lumitrac, Greiner), Already Containing 0.51 of Test Compound. All Plates Were Covered with Gas Permeable Seals and Incubated at $37^{\circ} \mathrm{C}, 5 \% \mathrm{CO}_{2}$ for 48 Hours. After 48 Hours, the Assay Plate Was Removed from the Incubator and Left to Cool to Room Temperature for 15 - 30 mins. Medium Was Removed from the Wells and $5 \mu \mathrm{l}$ Lysis Buffer (Renilla Luciferase Assay Kit, Promega) Was Added to Each Well. The Plate Was Incubated at Room Temperature on a Rocker for 15 mins then $15 \mu \mathrm{l}$ Assay Substrate was Added to Each Well. Luminescence Was Read Immediately Using an EnVision Plate Reader.

[15] Details of the Biochemical Assays and compound Screening within Them Will Be Described Elsewhere.

[16] J. Blagg, C. Mowbray, D. C. Pryde, G. Salmon, E. Schmid, D. Fairman and K. Beaumont, "Small, NonPeptide C5a Receptor Antagonists: Part 1," Bioorganic \& Medicinal Chemistry Letters, Vol. 18, 20, 2008, pp. 56015604. doi:10.1016/i.bmcl.2008.08.106

[17] J. Blagg, C. Mowbray, D. Pryde, G. Salmon, D. Fairman, E. Schmid and K. Beaumont, "Small, Non-Peptide C5a Receptor Antagonists: Part 2," Bioorganic \& Medicinal Chemistry Letters, Vol. 18, No. 20, 2008, pp. 5605-5608. doi:10.1016/j.bmcl.2008.08.101

[18] M. P. Edwards and D. A. Price, "Chapter 23-Role of Physicochemical Properties and Ligand Lipophilicity Efficiency in Addressing Drug Safety Risks," Annual Reports in Medicinal Chemistry, Vol. 45, 2010, pp. 380-391. doi:10.1016/S0065-7743(10)45023-X

[19] 5-(2-Thienyl)- $N$-(2-Thienylmethyl)-7-(Trifluoromethyl)-p
yrAzolo[1,5-a]Pyrimidine-2-Carboxamide, CAS Registry Number 314049-21-3.

[20] For the Weak Inhibition of Myc/Max Transcription Factors by Structurally Similar Compounds See A. Kiessling, B. Sperl, A. Hollis, D. Eick and T. Berg, "Selective Inhibition of c-Myc/Max Dimerization and DNA Binding by Small Molecules," Chemistry \& Biology, Vol. 13, No. 7, 2006, pp. 749-751. doi:10.1016/j.chembiol.2006.05.011

[21] S. K. Chunduru, C. A. Benetatos, T. J. Nitz and T. R. Bailey, "Compounds, Compositions and Methods for Treatment and Prophylaxis of Hepatitis C Viral Infections and Associated Diseases," US Patent No. US2004039533, 2005.

[22] G. Koutsoudakis, A. Kaul, E. Steinmann, S. Kallis, V. Lohmann, T. Pietschmann and R. Bartenschlager, "Characterization of the Early Steps of Hepatitis C Virus Infection by Using Luciferase Reporter Viruses," Journal of Virology, Vol. 80, No. 11, 2006, pp. 5308-5320. doi:10.1128/JVI.02460-05

[23] B. Feng, J. B. Mills, R. E. Davidson, R. J. Mireles, J. S. Janiszewski, M. D. Troutman and S. M. de Morais, "In Vitro P-Glycoprotein Assays to Predict the in Vivo Interactions of P-Glycoprotein with Drugs in the Central Nervous System," Drug Metabolism and Disposition, Vol. 36, No. 2, 2008, pp. 268-275. doi:10.1124/dmd.107.017434

[24] G. Allan, J. Davis, M. Dickins, I. Gardner, T. Jenkins, H. Jones, R. Webster and H. Westgate, "Pre-Clinical Pharmacokinetics of UK-453,061, a Novel Non-Nucleoside Reverse Transcriptase Inhibitor (NNRTI), and Use of in Silico Physiologically Based Prediction Tools to Predict the Oral Pharmacokinetics of UK-453,061 in Man," Xenobiotica, Vol. 38, No. 6, 2008, pp. 620-640. doi: 10.1080/00498250802069088

[25] K. Finlayson, L. Turnbull, C. T. January, J. Sharkey and J. S. Kelly, "[ $\left.{ }^{3} \mathrm{H}\right]$ Dofetilide Binding to HERG Transfected Membranes: A Potential High Throughput Preclinical Screen," European Journal of Pharmacology, Vol. 430, No. 1, 2001, pp. 147-148. doi:10.1016/S0014-2999(01)01362-0 